\title{
Sleep Researchers are Studying Addiction but Don't Know It
}

\author{
Harold W Gordon* \\ Division of Epidemiology, National Institute on Drug Abuse, USA
}

*Corresponding author: Harold W Gordon, Ph.D., Epidemiology Research Branch, Division of Epidemiology, Services and Prevention Research (DESPR), National Institute on Drug Abuse, The Neuroscience Center, Room 51516001 Executive Boulevard, Bethesda, MD 20892, USA.

Received Date: November 26, 2019

Published Date: December 05, 2019

\section{Commentary}

The purpose of this commentary is to survey published results of currently funded research grants from several institutes of the National Institutes of Health (NIH) on the topic of sleep. Grants to be included are those whose Aims are focused on the mission of their institutes but also could stimulate new research on the risks and consequences of addiction which is the mission of the National Institute on Drug Abuse. A recent survey [1] showed that drugs of addiction, while on board or during withdrawal, modify different aspects of the sleep architecture (e.g., onset, duration, slow wave sleep, REM sleep, etc.) and that the nature of these effects depended on the specific drug. Accordingly, those studying sleep for their own purposes may not know that they are also studying similar aspects of sleep relevant to addiction.

Alzheimer's Disease is one of the important diseases affected by sleep disturbances. A signature indication of Alzheimer's Disease is the presence of $\beta$-Amyloid in the brain. A grant to Spira (R01 AG 50507) from the National Institute on Aging aims to investigate whether poor sleep leads to $\beta$-Amyloid deposition. In a published study [2], increased $\beta$-Amyloid was seen in elderly participants who had reported daytime sleepiness (presumably a measure of disturbed sleep) at a baseline assessment 15 years earlier when the participants were age 60 . These results raise the question of whether individuals whose sleep is chronically disturbed by taking drugs will be at risk for Alzheimer's Disease.

Development of $\beta$-Amyloid deposition due to sleep disturbance is also the focus of a grant to Ju (K23 NS 89922) from the National Institute of Neurological Disorders and Stroke but in two types of participants: healthy individuals and those being treated for Obstructive Sleep Apnea (OSA). In the study of sleep-disturbed, neurologically normal individuals, presence of Amyloid- $\beta 40$ was strongly and significantly related to the disturbed slow wave activity [3]. In the clinical study [4], Amyloid- $\beta 40$ was reduced in correlation with improved sleep patients with OSA who had been tested before and after treatment by Positive Airwave Pressure (PAP) machines. Again, the relevance to addiction is whether disturbed slow wave sleep chronically observed in drug-taking individuals increases the risk of Amyloid- $\beta$ deposition and therefore Alzheimer's Disease.

Physical and mental conditions that are affected by drugs of abuse are also affected by sleep disturbances. Klerman has a grant (K24 HL105664) from National Heart, Lung, and Blood Institute to study the short- and long-term consequences of chronic sleep restriction on cognition and performance. While it is a not surprising result that participants in one study [5] were less vigilant, but it was disconcerting that they did not self-report a feeling of being less alert. Curiously, similar deficits in performance but with no feeling of less alertness was reported for cocaine addicts [6]. This disconnection between feelings of normal alertness in the presence of deficient cognitive performance from cocaine addiction suggests different neural systems are involved for each and worthy of further research.

While decline of cognitive function is well known in the aging population, a grant to Wilckens (K01 AG49879) focuses on whether improving sleep could moderate (improve) cognitive performance. Results of one study [7] showed that those who slept more had less of a memory impairment even in the presence of $\beta$-Amyloid than those with less sleep. If one can "artificially" enhance sleepespecially slow wave sleep-either by behavioral or pharmaceutical intervention [8], perhaps cognitive function can be improved as well. A similar focus on improving sleep as a partial treatment for addiction may prevent cognitive decline, or even reduce the risk of Alzheimer's Disease, in later life. 
Sleep disturbances have long been associated with more serious mental health disorders than cognitive decline, such as depression and schizophrenia. Current research funded by a grant to Ferrarelli (R01 MH113827) and a grant to Baran (K01 MH114012) from the National Institute of Mental Health are designed to study the underlying pathology of sleep spindles (EEG activity at $12-15 \mathrm{~Hz}$ ) which appear during Stage 2 (Non-REM) sleep and appear to be abnormal in patients with schizophrenia. Thus far, research in patients with schizophrenia has shown increased functional connectivity of the thalamus to cortical areas [9] and reduced spindle duration and density in frontal areas predicting negative symptoms [10]. Sleep spindles have not been studied as a potential neurobiological marker of addiction risk or of addiction consequences. However, as pointed out in our review [1], individuals withdrawing from virtually all drugs, including alcohol, have reduced Non-REM sleep. Especially notable is the persistent report of those withdrawing from marijuana who report nightmares and other psychotic episodes long after other symptoms have subsided. Accordingly, it is not unreasonable to hypothesize that potential deficits may be due to abnormal sleep spindles in those withdrawing from addiction.

Obesity and eating behaviors are both affected by short sleep; associated neural activity is being studied by a grant to Hart (R01 HL 92910)). Results of one study [11] showed increased neural activity in both the reward and inhibitory areas of the brain following food cues and short sleep. These are the same areas related to impulsive and reward behavior associated with addiction [12], suggesting neural mechanisms common to both.

Another physiological risk associated with sleep disturbances is cancer. As part of a grant (R01 CA207020) to Reynolds from the National Cancer Institute, the effect of chronotypes-whether individuals are morning "doves" or night "owls-were assessed as risk factors for cancer. In a study [13] of nearly 40,000 participants, those who reported to be "definite" evening types were significantly more likely to have cancer than those who reported to be "definite" morning types. As pointed out in the sleep/addiction review [1] "night owls" are also at greater risk for drug taking and addiction. These results suggest that eveningness chronotypes should be studied to learn what underlying factors are risks for addiction or diseases as serious as cancer.

As might be expected, research supported by the National Institute of Alcohol Abuse and Alcoholism most closely reflects the interests of the National Institute on Drug Abuse. Hasler has two grants (R01 AA26249; R01 AA25626) to study the underlying pathways-both neural and behavioral-that lead from sleep disturbance and specific chronotypes (morning- or eveningpreferring sleep times) to risk for alcohol and other drug use. Initial findings [14] from a large cohort study confirmed the observation that eveningness preference predicted greater alcohol use as well as greater marijuana use, both at baseline and at one-year follow-up. Controls indicate that this relationship is not due to social events which seems to imply the sleep characteristics and disturbances are related to a neurobiological risk factor. Continued research along these lines should provide evidence of underlying pathways beyond extensive alcohol use to addiction to other drugs.

Hasler also has a new grant (R01 DA44143) from the National Institute on Drug Abuse (NIDA) that intends to investigate the neurobehavioral pathways underlying a condition known as the delayed sleep phase (DSP) caused by circadian misalignment. The hypothesis is that DSP decreases impulse control and enhances reward sensitivity, both of which are risk factors for substance use. Another new grant to Finan (R01 DA48206) from NIDA will assess the interaction of sleep disturbance and low back pain. The hypothesis is that the "liking" effect and the "value" of taking opioids for treatment will be heightened in these individuals thereby increasing their risk for addiction. Results from these grants for these projects are not available for publication yet, but it is expected they will extend our knowledge of how underlying pathways of sleep neurobiology will also impact the neurobiology associated with addiction.

NIDA supports both basic and clinical research to address the opioid crisis with some focus on sleep. To date, through the Helping to End Addiction Long-term SM Initiative, or NIH HEAL Initiative SM, NIH has awarded eight grants in this area, including grants for basic mechanistic studies and clinical trial research. The awarded grants total approximately $\$ 24.9$ million. The projects will use various models and methods to understand the relationship between opioid use disorder (OUD) and sleep deficiency to improve outcomes for people with OUD. NIDA also supports work on the orexin system, known to be critical in sleep and wakefulness regulation, as a therapeutic target for addiction.

We have focused in this commentary on on-going, currently funded grants to investigators with a broad variety of expertise, all of whom are studying how sleep and sleep disturbances influence the particular disease or disorder relevant to their funding institute. Of course, there are considerably more on-going funded projects focused on sleep and circadian rhythm among the institutes of NIH than are highlighted here. The reason for selecting these studies is to demonstrate that their Aims have relevance for study of addiction. Indeed, the take-home lesson is that investigators who are doing sleep research for other diseases are also doing research relevant to addiction-but don't know it. Note: More information about the cited grants may be obtained from the NIH Project Reporter: https://projectreporter.nih.gov/

\section{Disclaimer}

The views and opinions expressed in this commentary are those of the author only and do not necessarily represent the views, official policy or position of the U.S. Department of Health and Human Services or any of its affiliated institutions or agencies.

\section{Acknowledgement}

None.

\section{Conflict of Interest}

No conflict of interest. 


\section{References}

1. Gordon HW (2019) Differential Effects of Addictive Drugs on Sleep and Sleep Stages. Journal of Addiction Research 3(2): 1-9

2. Spira AP, An Y, Wu MN, Owusu JT, Simonsick EM, et al. (2018) Excessive daytime sleepiness and napping in cognitively normal adults: associations with subsequent amyloid deposition by PiB PET. Sleep 41(10): 1-7.

3. Yo-E S, Ooms SHJ, Sutphen C, Macauley SL, Zangrilli MA, et al. (2017) Slow wave sleep disruption increases cerebrospinal fluid amyloid- $\beta$ levels. Brain 140(2): 2104-2111.

4. Ju YS, Zangrilli MA, Finn MB, Fagan AM, Holtzman DM (2018) Obstructive sleep apnea treatment, slow wave activity, and amyloid- $\beta$. Annals of Neurology 85: 291-295.

5. McHill AW, Hull JT, Wang W, Czeisler CA, Klerman EB (2018) Chronic sleep curtailment, even without extended (>15-h) wakefulness, degrades human vigilance performance. PNAS 115(23): 6070-6075.

6. Morgan PT, Pace-Schott EG, Sahul AH, Coric V, Stickgold R, Malison RT (2006) Sleep, sleep-dependent procedural learning an vigilance in chronic cocaine users: evidence for occult insomnia. Drug and Alcohol Dependence 82: 238-249.

7. Wilckens KA, Tudorascu DL, Snitz BE, Price JC, Aizenstein HJ, et al. (2018) Sleep moderates the relationship between amyloid beta and memory recall. Neurobiology of Aging 71: 142-148.
8. Wilckens KA, Ferrarelli F, Walker MP, Buysse DJ (2018) Slow-wave activity enhancement to improve cognition. Trends in Neuroscience 41(7): 470-482.

9. Baran B, Karahanoğlu FI, Mylonas D, Demanuele C, Vangel M, et al. (2019) Increased thalamocortical connectivity in schizophrenia correlates with sleep spindle deficits: Evidence for a common pathophysiology. Biological Psychiatry Cognitive Neuroscience and Neuroimaging 4(8): 706-714.

10. Kaskie RE, Graziano B, Ferrarelli F (2019) Topographic deficits in sleep spindle density and duration point to frontal thalamo-cortical dysfunctions in first-episode psychosis. Journal of Psychiatric Research 113: 39-44.

11. Demos KE, Sweet LH, Hart CN, McCaffery JM, Williams SE, et al. (2017) The effects of experimental manipulation of sleep duration on neural response to food cues. Sleep 40(11): 1-9.

12. Gordon HW (2016) Laterality of brain activation for risk factors of addiction. Current Drug Abuse Review 9: 1-18.

13. Hurley S, Goldberg D, Von Behren J, DeHart J, Wang S, et al. (2019) Chronotype and postmenopausal breast cancer risk among women in the California Teachers Study. Chronobiology International 27: 1-11.

14. Hasler BP, Franzen PL, de Zambotti M, Prouty D, Brown SA, et al. (2017) Eveningness and later sleep timing are associated with greater risk for alcohol and marijuana use in adolescences: Initial findings from the National Consortium on Alcohol and Neurodevelopment in Adolescence Study. Alcohol Clinical Experimental Research 41(6): 1154-1165. 